02

\title{
Химическое сжатие, структурная нестабильность и ИК-активные фононы в ряду редкоземельных аналогов францисита $\mathrm{Cu}_{3} \mathrm{RE}\left(\mathrm{SeO}_{3}\right)_{2} \mathrm{O}_{2} \mathrm{Cl}$
}

\author{
(C) Н.Н. Новикова ${ }^{1}$, В.А. Яковлев ${ }^{1}$, Е.С. Кузнецова ${ }^{2}$, П.С. Бердоносов ${ }^{2}$, С.А. Климин ${ }^{19}$ \\ ${ }^{1}$ Институт спектроскопии РАН, \\ 108840 Москва, Троицк, Россия \\ ${ }^{2}$ Московский гоударственный университет им. Ломоносова, химический фракультет, \\ 119991 Москва, Россия \\ I e-mail: klimin@isan.troitsk.ru
}

Поступила в редакцию 22.07.2021 г.

В окончательной редакции 22.07.2021 г.

Принята к публикации 05.08.2021 г.

Исследованы спектры нарушенного полного внутреннего отражения и пропускания серии франциситоподобных фаз с редкоземельными элементами $\mathrm{Cu}_{3} R E\left(\mathrm{SeO}_{3}\right)_{2} \mathrm{O}_{2} \mathrm{Cl}(R E-$ редкоземельный элемент, $R E=\mathrm{Nd}$, $\mathrm{Sm}, \mathrm{Eu}, \mathrm{Gd}, \mathrm{Dy}, \mathrm{Ho}, \mathrm{Tm}, \mathrm{Yb})$. Определены частоты ИК-активных фононов исследуемых кристаллов. В зависимостях частот фононов от ионного радиуса в серии изоструктурных соединений наблюдаются две тенденции: ужесточение частот за счет роста химического сжатия и смягчение частот колебаний, в которые вовлечены редкоземельные ионы, за счет увеличения массы конкретного редкоземельного иона. В кристалле $\mathrm{Cu}_{3} \mathrm{Dy}\left(\mathrm{SeO}_{3}\right)_{2} \mathrm{O}_{2} \mathrm{Cl}$ обнаружено аномальное смягчение низкочастотных фононов при низких температурах, связанное, по-видимому, со структурной нестабильностью соединения.

Ключевые слова: франциситоподобные фазы с редкоземельными элементами, оптическая спектроскопия, ИК-активные фононы, химическое сжатие.

DOI: $10.21883 / \mathrm{OS} .2022 .01 .51889 .22-21$

\section{Введение}

Фононный спектр несет важную информацию о среде. Он генетически связан с кристаллической структурой и поэтому является средством идентификации структуры и исследования фазовых переходов. Фононы в той или иной степени вовлечены во взаимодействия различных подсистем: решеточной, зарядовой, магнитной. Такие взаимодействия могут приводить как к образованию связанных мод, так и к необычному поведению фононов. В работах профессора Марины Поповой, юбилею которой посвящена данная работа, получены важные результаты по исследованиям мультифункциональных материалов при помощи спектроскопии колебательных состояний в области динамики решетки [1-7], структурных [8-10] и магнитоструктурных переходов [6,11-13], спин-фононных $[4,9,14-16]$, электрон-фононных [17-20] и межионных (давыдовских) [21] взаимодействий, магнито-диэлектрических [18] и изотопических [22] эффектов, связанных электрон-фононных $[14,19]$ и магнонфононных мод [3]. В данной работе, опираясь на опыт, полученный благодаря совместной с юбиляром работе, мы проводим первое исследование спектра инфракрасноактивных фононов новых редкоземельных соединений из семейства францисита.

Семейство редкоземельных аналогов минерала францисита с общей химической формулой $\mathrm{Cu}_{3} R E\left(\mathrm{SeO}_{3}\right)_{2} \mathrm{O}_{2} \mathrm{Cl}(R E-$ редкоземельный элемент,
Р3Э) было впервые синтезировано относительно недавно $[23,24]$ и привлекло внимание научной общественности благодаря интересным магнитным [25-31] и мультиферроидным [32] свойствам. Родоначальником семейства является одноименный природный минерал (францисит), оксоселенит-хлорид висмута-меди(II) $\mathrm{Cu}_{3} \mathrm{Bi}\left(\mathrm{SeO}_{3}\right)_{2} \mathrm{O}_{2} \mathrm{Cl}$ [33], обнаруженный впервые в 1987 г. на руднике Айрон Монарх (Австралия) служащим Гленом Фрэнсисом (Glyn Francis), имя которого запечатлелось в названии минерала. В минералогии подобного сочетания элементов ранее не встречалось [34]. В дальнейшем химики смогли синтезировать в лабораторных условиях как сам кристалл, отвечающий составу францисита, так и многочисленные производные от него с заменой хлора на другие галогены (бром и йод) [34], селена на теллур [35] и висмута на РЗЭ. Первым из синтетических франциситов с РЗЭ стал $\mathrm{Cu}_{3} \mathrm{Er}\left(\mathrm{SeO}_{3}\right)_{2} \mathrm{O}_{2} \mathrm{Cl}$ [36], a практически полная серия их была синтезирована в Московском государственном университете имени М.В. Ломоносова $[23,24]$. В результате большой набор изоструктурных соединений представляет собой модельный ряд для исследования систематических особенностей различных свойств, включая химические, структурные, магнитные, а также колебательные.

Кристаллическая структура минерала францисита была определена в 1990г. [33] и подтверждена позднее в ряде работ [37-39]. Он кристаллизуется в ромбической сингонии, пространственная группа Рmmn. Все 
известные к настоящему времени франциситоподобные фазы с РЗЭ также принадлежат указанной пространственной группе при комнатной температуре. Для них наблюдается практически монотонный рост трех параметров кристаллической решетки $a, b$ и $c$, а также объема элементарной ячейки $V$ с увеличением ионного радиуса РЗЭ [23]. На рис. 1 приведены фрагменты кристаллографической структуры францисита. Главными особенностями кристаллической решетки являются: 1 магнитные плоскости медной подрешетки, 2 - гексагональные каналы, содержащие атомы галогена, 3 молекулярные селенитные группы $\left(\mathrm{SeO}_{3}\right)^{2-}$. Магнитные плоскости устроены так, что ионы меди образуют так называемую решетку кагоме́ (см. проекцию $a b$, рис. 1), которая не является плоской, что хорошо видно на проекции $a c$, рис. 1. Фрустрации, характерные для решетки кагоме́, вследствие сильной конкуренции ферромагнитных и антиферромагнитных взаимодействий в медных плоскостях, не проявляются явно, однако их учет необходим и их роль в магнетизме францисита и его аналогов широко обсуждается в литературе [40-43].

Гексагональные каналы хорошо видны на проекции $a b$ (рис. 1). Внутри каналов находятся атомы хлора, а также неподелённые электронные пары селена, которые требуют определенного пространства. Каналы имеют разный размер в соединениях с различными трехзарядными ионами, при этом увеличение данного размера приводит к большей подвижности атомов галогена, которая, в частности, по данным работы [43], является причиной структурного фазового перехода, наблюдаемого в $\mathrm{Cu}_{3} \mathrm{Bi}\left(\mathrm{SeO}_{3}\right)_{2} \mathrm{O}_{2} \mathrm{Cl}$ при понижении температуры $\left(T_{C}=115 \mathrm{~K}\right)$. В этом смысле представляется интересным сравнение спектра фононов в серии соединений с разным размером каналов, так как фазовый переход в $\mathrm{Cu}_{3} \mathrm{Bi}\left(\mathrm{SeO}_{3}\right)_{2} \mathrm{O}_{2} \mathrm{Cl}$ сопровождается изменением спектра как ИК-, так и КРС-активных колебаний.

Молекулярные селенитные группы $\left(\mathrm{SeO}_{3}\right)^{2-}$ являются изолированными друг от друга комплексами с наиболее сильными межатомными связями. Такого рода комплексы, например $\left(\mathrm{BO}_{3}\right)^{2-}[9,14],\left(\mathrm{PO}_{4}\right)^{3-}[44]$, $\left(\mathrm{MoO}_{4}\right)^{2-}[10]$, так же как и $\left(\mathrm{SeO}_{3}\right)^{2-}$, помещенные в кристаллическое окружение, как правило, сохраняют колебательные частоты близкими к частотам свободного комплекса, что существенно облегчает анализ фононного спектра. Колебательные свойства селенитной группировки $\left(\mathrm{SeO}_{3}\right)^{2-}$ были ранее исследованы для ряда соединений [45-48]. Для анализа вклада группы селенита в фононный спектр франциситоподобных фаз необходимо провести корреляционный анализ для нормальных колебаний группы $\left(\mathrm{SeO}_{3}\right)^{2-}$ и сравнение с экспериментальными данными.

Фононный спектр франциситоподобных фаз подробно исследован только для висмутсодержащих соединений, которые можно вырастить в форме монокристаллов, в отличие от их аналогов с РЗЭ, которые удается синтезировать только в поликристаллической форме. В работе [48] исследованы спектры инфракрасного отражения
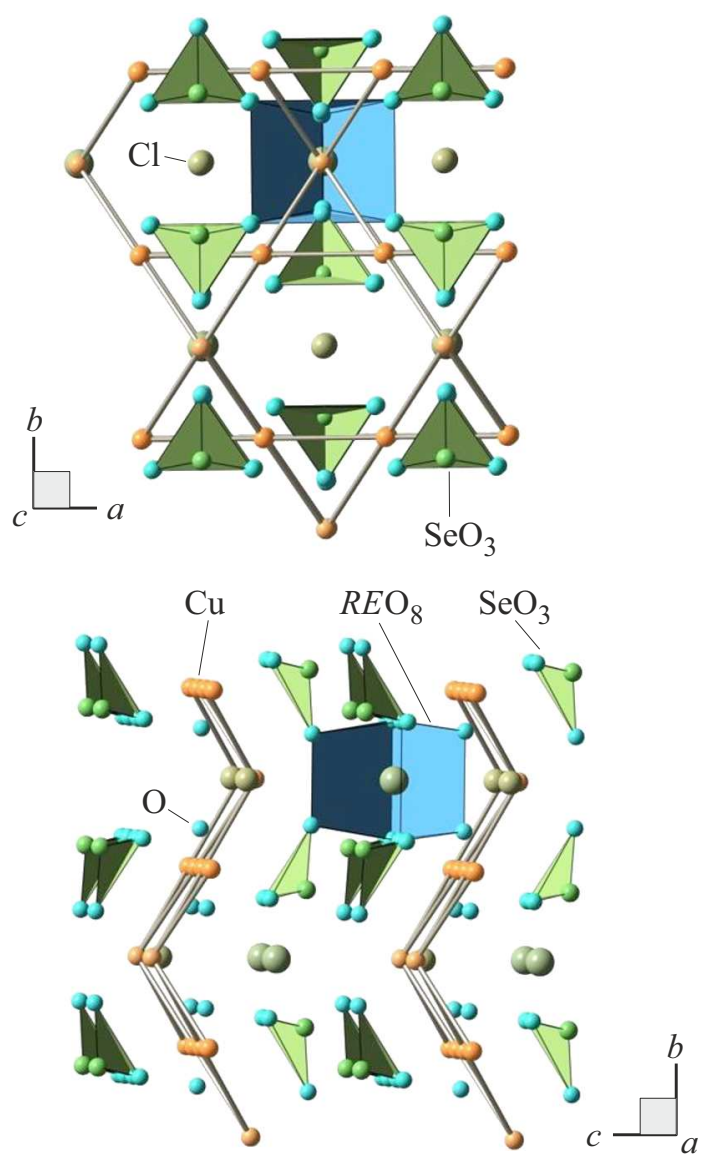

Рис. 1. Фрагмент кристаллической структуры франциситоподобных фаз $\mathrm{Cu}_{3} R E\left(\mathrm{SeO}_{3}\right)_{2} \mathrm{O}_{2} \mathrm{Cl}$, вид вдоль кристаллографической оси $c$ (верхняя панель) и оси $a$ (нижняя). Показаны треугольные искаженные пирамиды $\mathrm{SeO}_{3}$ и один полиэдр $R E \mathrm{O}_{8}$, Ближайшие атомы меди соединены связями, чтобы подчеркнуть наличие двумерных плоскостей и характер магнитной решетки в плоскости. Атомы хлора находятся внутри гексагональных каналов, направленных вдоль оси $c$.

в поляризованном свете и определены спектроскопические параметры ИК-активных фононов симметрии $B_{1 u}$, $B_{2 u}$ и $B_{3 и}$ для кристалла $\mathrm{Cu}_{3} \mathrm{Bi}\left(\mathrm{SeO}_{3}\right)_{2} \mathrm{O}_{2} \mathrm{Cl}$. В работе [32] проведено исследование спектров комбинационного рассеяния света (КРС) и дана информация по частотам КРС-активных фононов симметрии $A_{g}$ и $B_{1 g}$. Информация по фононному спектру франциситоподобных фаз, насколько нам известно, в литературе отсутствует.

В данной работе впервые проведено систематическое исследование инфракрасных (ИК) спектров в области фононов ряда синтетических аналогов францисита, содержащих Р3Э $\mathrm{Cu}_{3} R E\left(\mathrm{SeO}_{3}\right)_{2} \mathrm{O}_{2} \mathrm{Cl}(R E=\mathrm{Nd}, \mathrm{Sm}, \mathrm{Eu}$, $\mathrm{Gd}, \mathrm{Dy}, \mathrm{Ho}, \mathrm{Tm}, \mathrm{Yb})$ с целью определения параметров ИК-активных фононов и поиска закономерностей в поведении колебательных мод в ряду изоструктурных соединений с различными параметрами решетки и разными по массе ионами Р3Э. Для $\mathrm{Cu}_{3} \mathrm{Dy}\left(\mathrm{SeO}_{3}\right)_{2} \mathrm{O}_{2} \mathrm{Cl}$ проведе- 
но исследование температурных изменений параметров фононов с целью поиска низкотемпературных аномалий.

\section{Методы эксперимента}

Для синтеза исследуемых соединений использовали оксиды лантаноидов $\mathrm{Ln}_{2} \mathrm{O}_{3}(\mathrm{Ln}=\mathrm{Nd}, \mathrm{Sm}, \mathrm{Eu}, \mathrm{Gd}$, Dy, $\mathrm{Ho}, \mathrm{Tm}, \mathrm{Yb}$ ) (Гиредмет, Россия, не менее 99.99\%), оксид меди(II) $\mathrm{CuO}$ (степень чистоты 9-2), безводный хлорид меди(II) $\mathrm{CuCl}_{2}$ (Sigma-Aldrich $\geq 99.995 \%$ ) и оксид селена(IV) $\mathrm{SeO}_{2}$, предварительно полученный из селенистой кислоты $\mathrm{H}_{2} \mathrm{SeO}_{3}$ (квалификации XЧ) обезвоживанием в вакууме при температуре $70-90^{\circ} \mathrm{C}$ и возогнанный в токе осушенного воздуха с $\mathrm{NO}_{2}$.

Взятие навесок с $\mathrm{SeO}_{2}$ проводили в сухом боксе, продуваемом аргоном. Перечисленные выше прекурсоры были использованы для приготовления стехиометрических смесей в соответствии с уравнением реакции:

$5 \mathrm{CuO}+\mathrm{CuCl}_{2}+\mathrm{Ln}_{2} \mathrm{O}_{3}+4 \mathrm{SeO}_{2}=2 \mathrm{Cu}_{3} \mathrm{Ln}\left(\mathrm{SeO}_{3}\right)_{2} \mathrm{O}_{2} \mathrm{Cl}$.

Взвешивание прекурсоров проводили на весах Sartorius Gemp ${ }^{\text {lus }}$ с погрешностью $0.0002 \mathrm{~g}$ при общей массе навески для каждого состава не более $1 \mathrm{~g}$. Полученные для каждого состава смеси исходных веществ тщательно перетирали в агатовой ступке и помещали в кварцевые ампулы, которые затем отпаивали под вакуумом $\sim 10^{-2} \mathrm{~mm} \mathrm{Hg}$. После этого каждую из смесей отжигали в печи в следующем режиме: нагрев до $300^{\circ} \mathrm{C}$ в течение $12 \mathrm{~h}$, выдерживание при постоянной температуре в течение $24 \mathrm{~h}$, нагрев до $575^{\circ} \mathrm{C}$ в течение $6 \mathrm{~h}$, выдерживание при этой температуре в течение $72 \mathrm{~h}$.

В результате были получены порошкообразные соединения зеленого цвета с однородной окраской. Рентгенофазовый анализ, проведенный на дифрактометре STOE Stadi-P (источник $\mathrm{Cu} K_{\alpha 1}$ ), подтвердил однофазность полученных соединений. Рентгенограммы всех продуктов были схожи между собой и полностью индицировались в пространственной группе Ртmп. Параметры элементарных ячеек полностью соответствуют данным работы [23].

Для исследования ИК спектров полного внутреннего отражения была использована приставка НПВО с алмазной призмой, угол падения составлял $45^{\circ}$. Измерения проводились на фурье-спектрометре BRUKER IFS66 v/s в широком спектральном диапазоне от 50 до $2000 \mathrm{~cm}^{-1}$ в два приема: в дальнем ИК диапазоне от 50 до $550 \mathrm{~cm}^{-1}$ и в среднем ИК диапазоне от 400 до $5000 \mathrm{~cm}^{-1}$, спектральное разрешение составляло $4 \mathrm{~cm}^{-1}$. Источником излучения в обоих случаях являлся глобар, представляющий собой стержень из карбида кремния $\mathrm{SiC}$, разогретый до высоких температур. В длинноволновой спектральной области использовался лавсановый светоделитель с покрытием из германия и пироэлектрический приемник на основе ДЛАТГС (дейтерированный триглицинсульфат с примесью L- $\alpha$-аланина), в коротковолновой светоделитель из $\mathrm{KBr}$ и пироэлектрический приемник на основе ДТГС (дейтерированный триглицинсульфат
$\left.\left(\mathrm{NH}_{2} \mathrm{CH}_{2} \mathrm{OOH}\right)_{3} \cdot \mathrm{H}_{2} \mathrm{SO}_{4}\right)$. Для уменьшения шумов в области низких частот спектры были дополнительно измерены с использованием гелиевого болометра, при этом измерения проводились на фурье-спектрометре BRUKER IFS125HR.

Температурнозависимые спектры диффузного пропускания диспрозиевого аналога минерала францисита $\mathrm{Cu}_{3} \mathrm{Dy}\left(\mathrm{SeO}_{3}\right)_{2} \mathrm{O}_{2} \mathrm{Cl}$ были измерены на фурьеспектрометре BRUKER IFS125HR. Поликристаллический образец был растолчен в агатовой ступке, после чего была создана мелкодисперсная взвесь исследуемого соединения в спирте. Взвесь осаждалась на алмазную пластинку и после высыхания на поверхности алмаза образовывалась тонкая равномерная пленка. После этого алмаз с образовавшейся пленкой был плотно прижат с использованием индиевой прокладки к хладопроводу оптического гелиевого криостата CRYOMECH PT403 с полиэтиленовыми оптическими окнами.

\section{Результаты исследований и обсуждения}

Спектры нарушенного полного внутреннего отражения (НПВО) франциситоподобных фаз $\mathrm{Cu}_{3} R E\left(\mathrm{SeO}_{3}\right)_{2} \mathrm{O}_{2} \mathrm{Cl}(R E=\mathrm{Nd}, \mathrm{Sm}, \mathrm{Eu}, \mathrm{Gd}, \mathrm{Dy}, \mathrm{Ho}, \mathrm{Tm}$, $\mathrm{Yb})$ в широком спектральном диапазоне приведены на рис. 2. Спектры для восьми представленных соединений сходны, что и ожидалось для серии изоструктурных соединений. Этот факт также является косвенным подтверждением того, что, действительно, все соединения обладают одинаковой кристаллографической структурой.

Фактор-групповой анализ дает следующую формулу для оптических колебательных мод франциситов со структурой Рmmn [32,48]:

$$
\begin{aligned}
& \Gamma_{\mathrm{opt}}=14 B_{1 u}(E \| z)+14 B_{2 u}(E \| y)+11 B_{3 u}(E \| x) \\
& +12 A_{g}(x x, y y, z z)+6 B_{1 g}(x y, y x)+9 B_{2 g}(x z, z x) \\
& +12 B_{3 g}(x y, y x)+9 A_{1 u} .
\end{aligned}
$$

В скобках указана активность колебательных мод: по направлению электрического поля $\mathbf{E}$ световой волны для ИК-активных фононов и по направлению электрического поля падающего и анализируемого излучения для КРСактивных мод. Моды симметрии $A_{1 u}$ оптически неактивны.

Согласно формуле (2), суммарно в кристаллах исследуемых фаз структуры Pmmn должно наблюдаться 39 ИК-активных мод. Однако, как следует из данных работы [48], в кристалле $\mathrm{Cu}_{3} \mathrm{Bi}\left(\mathrm{SeO}_{3}\right)_{2} \mathrm{O}_{2} \mathrm{Cl}$ ИК моды сильно перекрываются: некоторые частоты колебаний разной симметрии имеют очень близкие значения. Более того, авторы указанной работы, зарегистрировав в двух поляризациях количественно ровно столько ИКактивных мод, сколько ожидается по фактор-групповому анализу, в третьей поляризации из 14 ИК-активных мод симметрии $B_{1 u}$ смогли обнаружить только 11 , повидимому, также вследствие близости частот некоторых 


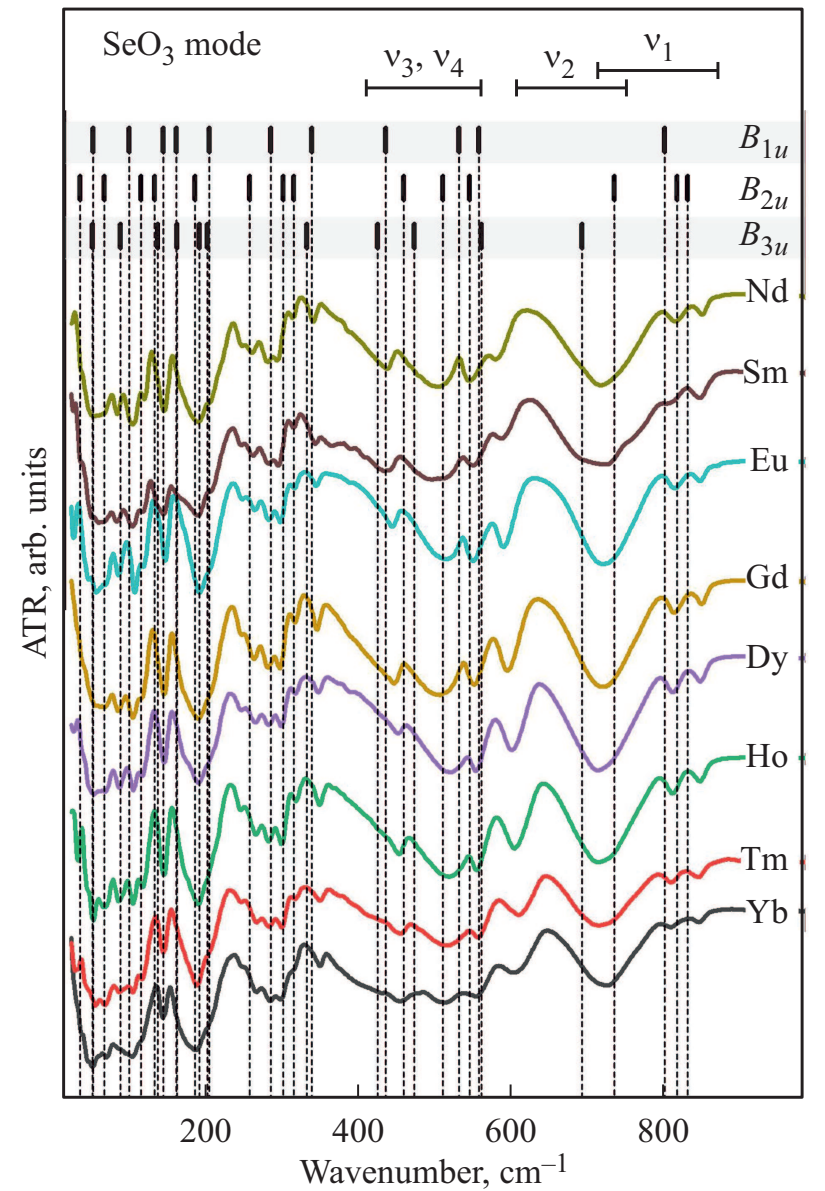

Рис. 2. Экспериментальные спектры НПВО для редкоземельных аналогов францисита $\mathrm{Cu}_{3} R E\left(\mathrm{SeO}_{3}\right)_{2} \mathrm{O}_{2} \mathrm{Cl}(R E=\mathrm{Nd}, \mathrm{Sm}$, $\mathrm{Eu}, \mathrm{Gd}, \mathrm{Dy}, \mathrm{Ho}, \mathrm{Tm}, \mathrm{Yb})$ в широкой спектральной области, измеренные при комнатной температуре. Спектры произвольно сдвинуты для наглядности по оси ординат. Черточки с ниспадающими вертикальными штриховыми линиями соответствуют ТО-частотам для кристалла $\mathrm{Cu}_{3} \mathrm{Bi}\left(\mathrm{SeO}_{3}\right)_{2} \mathrm{O}_{2} \mathrm{Cl}$ [48]. Сверху указаны интервалы частот для внутренних колебаний молекулярных комплексов $\left(\mathrm{SeO}_{3}\right)^{2-}$.

мод. Кроме этого, подчеркнем, что данные в указанной работе получены при гелиевой температуре $(7 \mathrm{~K})$, когда затухание фононных мод минимально. При комнатной температуре вследствие уширения фононов перекрытие растет. Действительно, сравнение спектров пропускания кристалла $\mathrm{Cu}_{3} \mathrm{Dy}\left(\mathrm{SeO}_{3}\right)_{2} \mathrm{O}_{2} \mathrm{Cl}$, измеренных при комнатной $(300 \mathrm{~K})$ и низкой $(15 \mathrm{~K})$ температурах, наглядно показывает, что вследствие температурного уширения и перекрывания многие линии (отмечены стрелками на рис. $3, a$ ) становятся неразрешимыми при высоких температурах. При таком большом количестве фононов и невозможности измерить поляризованные спектры на поликристаллических образцах можно предположить, что в редкоземельных аналогах францисита при комнатной температуре количество зарегистрированных ИК мод будет значительно меньше числа мод, ожидаемого по теоретико-групповому анализу.
В измеренных при комнатной температуре спектрах НПВО в зависимости от соединения мы смогли зафиксировать до 25 пиков, включая „плечи“ на крыльях сильных линий поглощения, частоты которых сведены в таблицу, в которой для сравнения приведены данные по ИК-активным фононам для $\mathrm{Cu}_{3} \mathrm{Bi}\left(\mathrm{SeO}_{3}\right)_{2} \mathrm{O}_{2} \mathrm{Cl}$ из работы [48]. Для наглядности сравнение с данными для монокристалла $\mathrm{Cu}_{3} \mathrm{Bi}\left(\mathrm{SeO}_{3}\right)_{2} \mathrm{O}_{2} \mathrm{Cl}$, доступными в литературе, приведено на рис. 2. Наблюдается неплохое соответствие полученных данных с литературными, особенно если принять во внимание сдвиги частот, обусловленные как разницей параметров кристаллической решетки, так и массой трехзарядного иона РЗЭ.

Отметим также общеизвестный факт, что минимумы в спектрах НПВО находятся вблизи ТО-частот, но не совпадают с ними. Для демонстрации этого факта мы провели моделирование спектров пропускания и спектров НПВО с использованием оптических параметров, полученных в работе [48]. Диэлектрическая проницаемость определяется дисперсионным соотношением через сумму вкладов $N$ независимых затухающих осцилляторов:

$$
\varepsilon(\omega)=\varepsilon_{\infty}+\sum_{j=0}^{N} \frac{S_{j} \omega_{j}^{2}}{\omega_{j}^{2}-\omega^{2}+i \gamma_{j} \omega},
$$

где $\varepsilon_{\infty}$ - диэлектрическая проницаемость на высоких частотах, $\omega_{j}, S_{j}$ и $\gamma_{j}-$ частота, сила осциллятора и полуширина для $j$-го фонона. Вычисления спектров НПВО и спектров пропускания были проведены с использованием программы SCOUT [49]. На рис. 3, $b$ сравниваются рассчитанные спектры НПВО и пропускания для фононов симметрии $B_{1 u}$ в ограниченной спектральной области. Видно, что для слабоинтенсивных линий положение минимумов пиков пропускания практически совпадает с ТО-частотами. Для более интенсивных линий в спектре НПВО наблюдаются незначительные смещения.

Отметим, что в спектрах на рис. 3 наблюдаются несколько групп линий, разделенных небольшими интервалами. Наличие групп линий связано с возможностью разделения фононов по типам: на внутренние колебания молекулярной группы $\left(\mathrm{SeO}_{3}\right)^{2-}$ и внешние по отношению к ней.

Свободный комплекс $\left(\mathrm{SeO}_{3}\right)^{2-}$ обладает симметрией $C_{3 v}$ и имеет 4 нормальных колебания: невырожденные $v_{1}$ и $v_{2}$ симметрии $A_{1}$ и вырожденные $v_{3}$ и $v_{4}(E)$ [50]. Мы следуем именно такой системе обозначений, хотя в литературе встречаются разночтения. Часто колебание $v_{1}$ называют симметричным $\left(v_{s}\right), v_{2}-$ асимметричным $\left(v_{a s}\right)$, а $v_{3}$ и $v_{4}$ - валентными $(\delta-$ bending). Частота каждого из нормальных колебаний немного изменяется, когда молекулярная группа находится в конкретной матрице. Основываясь на работах [45-47], в которых исследовались частоты колебаний молекулярной группы $\left(\mathrm{SeO}_{3}\right)^{2-}$ в разных кристаллах, мы определили примерный интервал частот для каждого из колебаний, показанный сверху на рис. 2. Внутренние колебания группы $\left(\mathrm{SeO}_{3}\right)^{2-}$ занимают наиболее высокочастотный 
Частоты ИК-активных фононов $\left(\right.$ в $\left.\mathrm{cm}^{-1}\right)$ фаз со структурой францисита $\mathrm{Cu}_{3} R E\left(\mathrm{SeO}_{3}\right)_{2} \mathrm{O}_{2} \mathrm{Cl}$ и их соотнесение с ТО-фононами в $\mathrm{Cu}_{3} \mathrm{Bi}\left(\mathrm{SeO}_{3}\right)_{2} \mathrm{O}_{2} \mathrm{Cl}[48]$

\begin{tabular}{|c|c|c|c|c|c|c|c|c|c|}
\hline \multicolumn{9}{|c|}{ Данная работа } & \multirow{2}{*}{$\frac{[48]}{\mathrm{Bi}}$} \\
\hline $\mathrm{Yb}$ & $\mathrm{Tm}$ & Ho & Dy & $\mathrm{Gd}$ & $\mathrm{Eu}$ & $\mathrm{Sm}$ & $\mathrm{Nd}$ & Комментарий & \\
\hline & 32 & 32.6 & & & & 31.1 & & Плечо & $36.3\left(B_{2 u}\right)$ \\
\hline 48.7 & 45.5 & 45.1 & 48.4 & 40.7 & 42.2 & 42.7 & 41.4 & & $52.8\left(B_{3 u}\right)$ \\
\hline- & 56.8 & 54.1 & - & & 56.5 & 56.7 & 56.1 & & $53.2\left(B_{1 u}\right)$ \\
\hline 70.4 & 69.5 & 68.4 & 69.1 & 69.2 & 69.5 & 69.2 & 69.1 & & $68.3\left(B_{2 u}\right)$ \\
\hline 89.3 & 86.9 & 85.6 & 85.7 & 84.7 & 85.5 & 84.3 & 84.4 & & $89\left(B_{3 u}\right)$ \\
\hline 104.5 & 104.9 & 105 & 105.3 & 104.8 & 106.8 & 104.8 & 104.5 & & $99.8\left(B_{1 u}\right)$ \\
\hline 116.6 & 116.2 & 116.1 & 116.7 & 117.2 & 118.7 & 118.6 & 120.1 & Плечо & $115.2\left(B_{2 u}\right)$ \\
\hline 143.7 & 144 & 144.1 & 144.9 & 145.3 & 145.9 & 145.1 & 145.2 & & $\begin{array}{l}133.5\left(B_{2 u}\right)+ \\
137.6\left(B_{3 u}\right)+144.8\left(B_{1 u}\right)\end{array}$ \\
\hline 176.4 & 175.5 & 172.3 & 173.4 & 170.9 & 170.6 & 167.9 & 169 & Плечо & $161.9\left(B_{3 u}\right)+161.5\left(B_{1 u}\right)$ \\
\hline 187.1 & 187 & 188 & 188.2 & 189 & 190 & 190 & 190.5 & & $185.8\left(B_{2 u}\right)+191.6\left(B_{3 u}\right)$ \\
\hline 201.6 & 202.6 & 202 & 202 & 207 & 205.7 & 204.9 & 204 & Плечо & $202.1\left(B_{3 u}\right)+204\left(B_{1 u}\right)$ \\
\hline 245 & 244.8 & 244.7 & 245.1 & 245.8 & 246.5 & 245.4 & 246.1 & & $256.9\left(B_{2 u}\right)$ \\
\hline 266 & 265 & 263.2 & 263 & 261.8 & 262.9 & 260.5 & 260.1 & & $-{ }^{\prime \prime}-$ \\
\hline 282 & 281.7 & 281 & 280.5 & 280.5 & 280.9 & 280.2 & 279.7 & & $284.4\left(B_{1 u}\right)$ \\
\hline 298.1 & 297.9 & 297.5 & 296.5 & 295.3 & 296 & 292.9 & 291.5 & Плечо & $300.3\left(B_{2 u}\right)$ \\
\hline 316.6 & 316.5 & 316.3 & 316.2 & 315.9 & 314.6 & 314.3 & 312.9 & & $313.9\left(B_{2 u}\right)$ \\
\hline 348.5 & 348.1 & 346.9 & 345.9 & 344 & 343 & 341.1 & 339.2 & Плечо & $331.1\left(B_{3 u}\right)+337.6\left(B_{1 u}\right)$ \\
\hline 426.4 & 428.4 & 426.8 & 425.9 & 423.6 & 421.2 & 424 & 418.3 & & $422.9\left(B_{2 u}\right)$ \\
\hline 454 & 451.8 & 448.8 & 447.1 & 444.3 & 440.1 & 438 & 433.4 & & $456.3\left(B_{2 u}\right)+433.5\left(B_{1 u}\right)$ \\
\hline 511 & 512 & 513 & 514 & 507 & 508 & 502 & 500 & Широкая & $470.2\left(B_{3 u}\right)+507\left(B_{2 u}\right)$ \\
\hline 553 & 553 & 550.8 & 549.9 & 546.9 & 544.9 & 545.8 & 538.7 & & $528.4\left(B_{1 u}\right)$ \\
\hline 604 & 605.4 & 600.1 & 596.6 & 591.2 & 586.4 & 584.5 & 576.9 & & $\begin{array}{l}542.3\left(B_{2 u}\right)+ \\
557.3\left(B_{3 u}\right)+554.4\left(B_{1 u}\right)\end{array}$ \\
\hline 717 & 712 & 714 & 712 & 716 & 715.5 & 718.6 & 717 & & $688.2\left(B_{3 u}\right)+730\left(B_{2 u}\right)$ \\
\hline 803.9 & 804.2 & 805.3 & 806.6 & 807.4 & 808 & 808 & 809 & & $811.3\left(B_{2 u}\right)+794.9\left(B_{1 u}\right)$ \\
\hline 840 & 839.8 & 840.8 & 841.3 & 842.5 & 842.4 & 842.3 & 843.7 & & $825\left(B_{2 u}\right)$ \\
\hline
\end{tabular}

интервал в фононном спектре франциситов, при этом самые высокие частоты имеют колебания $\nu_{1}$ и $\nu_{2}$.

Для определения количества мод, соответствующих внутренним колебаниям комплекса селенита $\left(\mathrm{SeO}_{3}\right)^{2-}$, в каждом из представлений кристалла мы провели корреляционный анализ. На рис. 4 приведена часть этого анализа, относящаяся к внутренним колебаниям $\left(\mathrm{SeO}_{3}\right)^{2-}$. Элементарная ячейка кристаллов францисита структуры Рmmn содержит 2 формульные единицы и соответственно 4 молекулярные группы $\mathrm{SeO}_{3}$. В кристалле симметрия свободной молекулы $C_{3 v}$ понижается до $C_{s}$. Это искажение небольшое: если в неискаженной молекуле три расстояния $\mathrm{Se}-\mathrm{O}$ одинаковы, то в кристалле они слегка отличаются: одно расстояние (вычисленное для структуры $\mathrm{Cu}_{3} \mathrm{Bi}\left(\mathrm{SeO}_{3}\right)_{2} \mathrm{O}_{2} \mathrm{Cl}$ [34]) составляет $1.73 \AA$, а два других равны между собой (для симметрии $C_{s}$ ) и составляют $1.69 \AA$. Как следует из корреляционной схемы, среди ИК-активных фононов (2) находятся по две моды для самых высокочастотных колебаний $v_{1}$ и $v_{2}$, что неплохо согласуется с полученными в данной работе спектрами. В спектральной области частоты $v_{1}$, действительно, в спектрах всех восьми соединений наблюдаются две полосы поглощения, в то время как для колебания $v_{2}$ наблюдается только одна полоса, ко- торая, по-видимому, представляет собой общий контур для двух перекрывающихся полос. Полосы поглощения для полносимметричного колебания $v_{1}$ различных молекулярных групп малоинтенсивны (примеры можно найти в работах, например, [14,44]) вследствие того, что в свободной молекуле полносимметричные колебания не создают дипольного момента, необходимого для электродипольного взаимодействия со световой волной. Небольшой дипольный момент нормальных колебаний, коррелирующих с колебанием $v_{1}$, появляется, когда молекула находится в кристалле и ее симметрия понижена.

На рис. 5 приведены спектры НПВО (в узкой спектральной области) франциситоподобных фаз, исследуемых в данной работе. Спектры на рисунке расположены таким образом, что табличный номер иона РЗЭ, входящего в соединение, растет плавно при рассмотрении спектров рис. 5 сверху вниз. Значение ионного радиуса было взято из таблиц Шеннона [51,52], данные для восьмикратной координации, которая имеет место в структуре аналогов францисита, содержащих ионы РЗЭ.

Пики поглощения на рис. 5 также плавно смещаются от соединения к соединению, причем характер этих смещений разный. Так, например, фононные моды вблизи 350,450 и $500 \mathrm{~cm}^{-1}$ смещаются в высокочастотную 


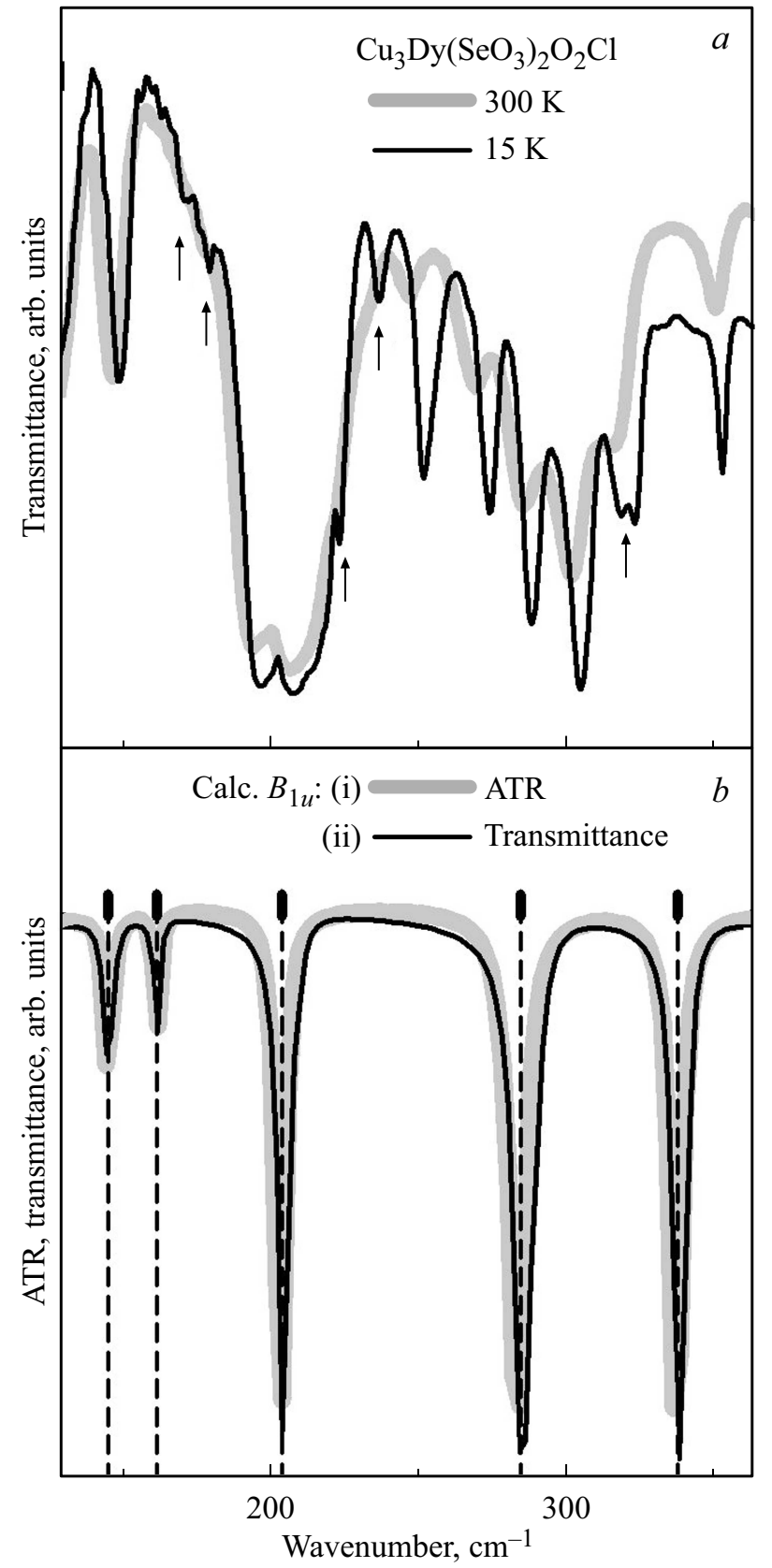

Pис. 3. (a) Диффузные спектры пропускания поликристаллического $\mathrm{Cu}_{3} \mathrm{Dy}\left(\mathrm{SeO}_{3}\right)_{2} \mathrm{O}_{2} \mathrm{Cl}$ при комнатной и низкой $(15 \mathrm{~K})$ температурах. (b) Рассчитанные спектры НПВО (серая толстая линия) и пропускания (черная тонкая) кристалла $\mathrm{Cu}_{3} \mathrm{Bi}\left(\mathrm{SeO}_{3}\right)_{2} \mathrm{O}_{2} \mathrm{Cl}$.

сторону с уменьшением ионного радиуса, в то время как моды вблизи 140 и $170 \mathrm{~cm}^{-1}$ смещаются в низкочастотную область.

Данные смещения можно объяснить, учитывая, что, во-первых, параметры элементарной ячейки также плавно меняются в зависимости от ионного радиуса редкоземельного иона и, во-вторых, масса иона РЗЭ в ряду исследуемых соединений изменяется на $\sim 20 \%$.

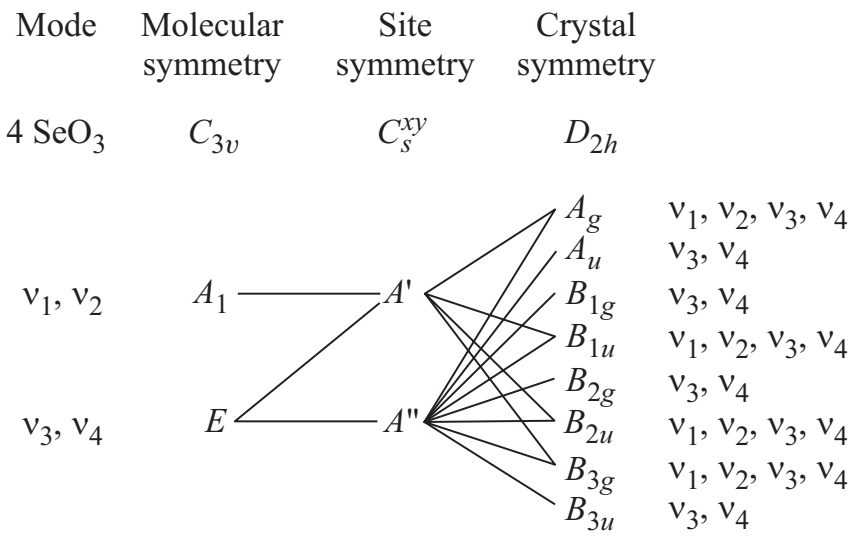

Рис. 4. Корреляции внутренних нормальных колебаний группы $\left(\mathrm{SeO}_{3}\right)^{2-}$ в кристаллах тетраэдрической симметрии $D_{2 h}$.

Уменьшение размера элементарной ячейки при уменьшении ионного радиуса РЗЭ можно рассматривать как явление „химического сжатия“. Ожидается, что химические связи „в среднем“ будут становиться более жесткими при сжатии ячейки, что должно приводить соответственно к росту („ужесточению“) колебательных частот, что легко качественно понять для системы двух грузиков, связанных пружиной, в соответствии со следующим широко известным соотношением:

$$
v=\sqrt{\frac{k}{m}},
$$

где $v$ - частота колебаний системы, $k$ - жесткость пружины, $m$ - приведенная масса. Согласно уравнению (4), утяжеление атома, участвующего в данном колебании, должно приводить к уменьшению частоты колебания. Таким образом, те колебательные моды, в которых участвуют ионы РЗЭ, должны также быть зависимыми от соединения. Поскольку РЗЭ - самый тяжелый из химических элементов, входящих в соединение, то и нормальные колебания, в которых участвует редкая земля, будут иметь низкие частоты. Заметим, что в ряду редкоземельных ионов, имеющих отношение к данному исследованию, при движении от $\mathrm{Yb}$ к $\mathrm{Nd}$ ионный радиус, так же как и параметры ячейки, увеличивается, а масса иона уменьшается. Таким образом, в рассматриваемой последовательности ожидается, что частота будет уменьшаться за счет эффекта химического сжатия и увеличиваться за счет влияния массы иона.

На рис. 6 приведены зависимости от ионного радиуса для нормированных параметров решетки и для нормированных частот (энергий) некоторых нормальных колебаний из частотного диапазона выше $300 \mathrm{~cm}^{-1}$. Приведенные на графике значения брались как отношение частоты или параметра решетки в данном соединении к соответствующим значениям для иттербиевого францисита. Наблюдается небольшая нелинейность параметров решетки $b$ и $c$, имеющая некоторое проявление в зависимостях частот. В целом частоты практически 


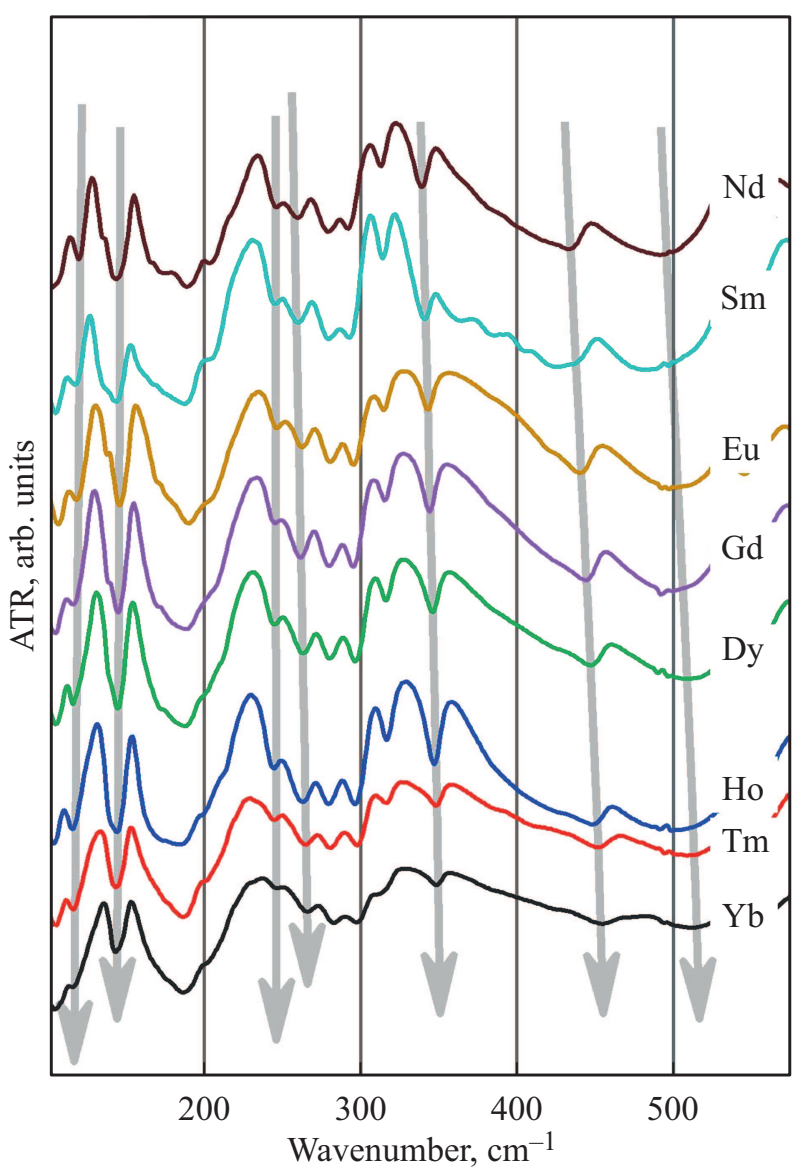

Рис. 5. Экспериментальные спектры НПВО для франциситоподобных фаз $\mathrm{Cu}_{3} R E\left(\mathrm{SeO}_{3}\right)_{2} \mathrm{O}_{2} \mathrm{Cl}(R E=\mathrm{Nd}, \mathrm{Sm}, \mathrm{Eu}, \mathrm{Gd}$, $\mathrm{Dy}, \mathrm{Ho}, \mathrm{Tm}, \mathrm{Yb})$ в низкочастотной спектральной области при комнатной температуре. Спектры произвольно сдвинуты для наглядности по оси ординат. Стрелки показывают систематический сдвиг спектральных линий в зависимости от ионного радиуса редкоземельного иона.

линейно зависят от ионного радиуса, что укладывается в модель химического сжатия: чем меньше ионный радиус Р3Э, тем меньше параметры решетки, плотнее упаковка, жестче связи и соответственно выше частоты колебаний.

На рис. 7 приведены нормированные частоты для некоторых низкочастотных нормальных колебаний франциситоподобных фаз $\mathrm{Cu}_{3} R E\left(\mathrm{SeO}_{3}\right)_{2} \mathrm{O}_{2} \mathrm{Cl}$. Данные зависимости частот от ионного радиуса имеют совсем другой характер по сравнению с зависимостями для мод, приведенными на рис. 6. В целом частота каждой из представленных мод растет с увеличением ионного радиуса. Мы предполагаем, что данные моды связаны с колебаниями, в которых участвует редкоземельный ион. Кроме частот, на рис. 7 показана зависимость нормированного корня квадратного от обратной массы иона Р3Э $\left(\sqrt{M_{\mathrm{Yb}} / M_{R E}}\right)$. На частоты колебательных мод с участием редкоземельного иона влияют оба фактора, как фактор „массы“, так и фактор химического сжатия, вследствие этого частота колебания растет при увеличе-

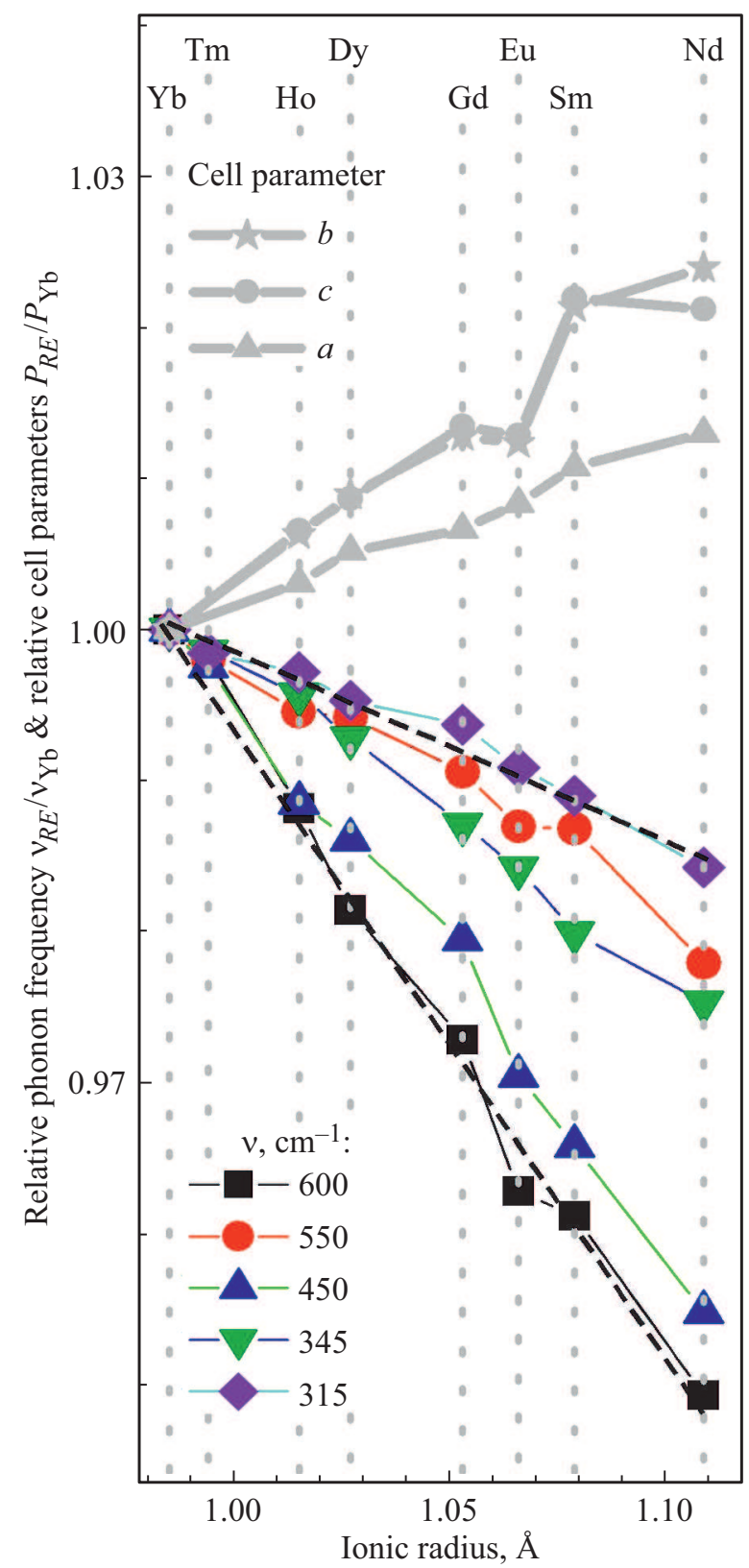

Рис. 6. Нормированные параметры решетки (серые символы) и частоты (цветные символы) некоторых колебательных мод франциситоподобных фаз $\mathrm{Cu}_{3} R E\left(\mathrm{SeO}_{3}\right)_{2} \mathrm{O}_{2} \mathrm{Cl}$ в зависимости от ионного радиуса иона редкоземельного элемента.

нии ионного радиуса в значительно меньшей мере, чем могла бы расти при учете только массы участвующего в колебании иона. Таким образом, из анализа частотного поведения фононов в зависимости от ионного радиуса нам удалось выделить колебательные моды, генетически связанные с движением редкоземельного иона.

Мы также провели исследование спектров диффузного пропускания кристалла $\mathrm{Cu}_{3} \mathrm{Dy}\left(\mathrm{SeO}_{3}\right)_{2} \mathrm{O}_{2} \mathrm{Cl}$ при охлаждении образца. На рис. 8 показаны спектры пропускания при трех различных температурах: 300, 80 и $7 \mathrm{~K}$. Были выбраны два фонона, проявляющих температур- 


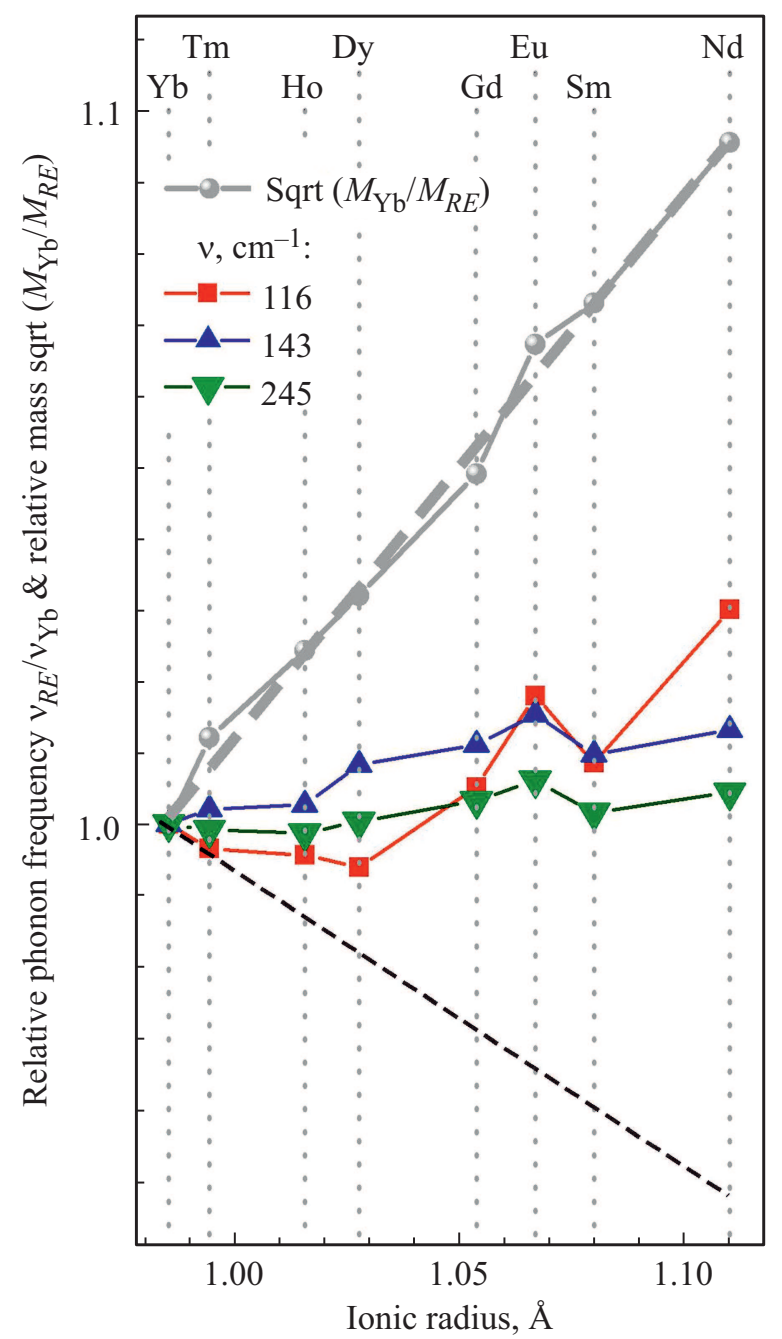

Рис. 7. Нормированные частоты некоторых низкочастотных колебательных мод (цветные треугольники и квадраты) франциситоподобных фаз $\mathrm{Cu}_{3} R E\left(\mathrm{SeO}_{3}\right)_{2} \mathrm{O}_{2} \mathrm{Cl}$ и корень квадратный обратной массы редкоземельного иона (серые шарики и линии) в зависимости от ионного радиуса редкоземельного иона. Черной штриховой линией для сравнения обозначено поведение моды $600 \mathrm{~cm}^{-1}$.

ное поведение разного характера. Фонон с частотой вблизи $350 \mathrm{~cm}^{-1}$ демонстрирует нормальное поведение. Во-первых, частота фонона растет с понижением температуры вследствие упоминавшегося выше эффекта химического сжатия. Кристалл при охлаждении уменьшается в размерах, межионные связи становятся более жесткими, что приводит к повышению колебательных частот. Во-вторых, полуширина данного фонона уменьшается с понижением температуры. Такое поведение является нормальным, так как при охлаждении уменьшается количество процессов распада данного фононного состояния. В частности, вследствие уменьшения заселенности колебательных состояний при низкой температуре остаются только релаксационные процессы распада. Отметим, что практически все фононы в измеренном

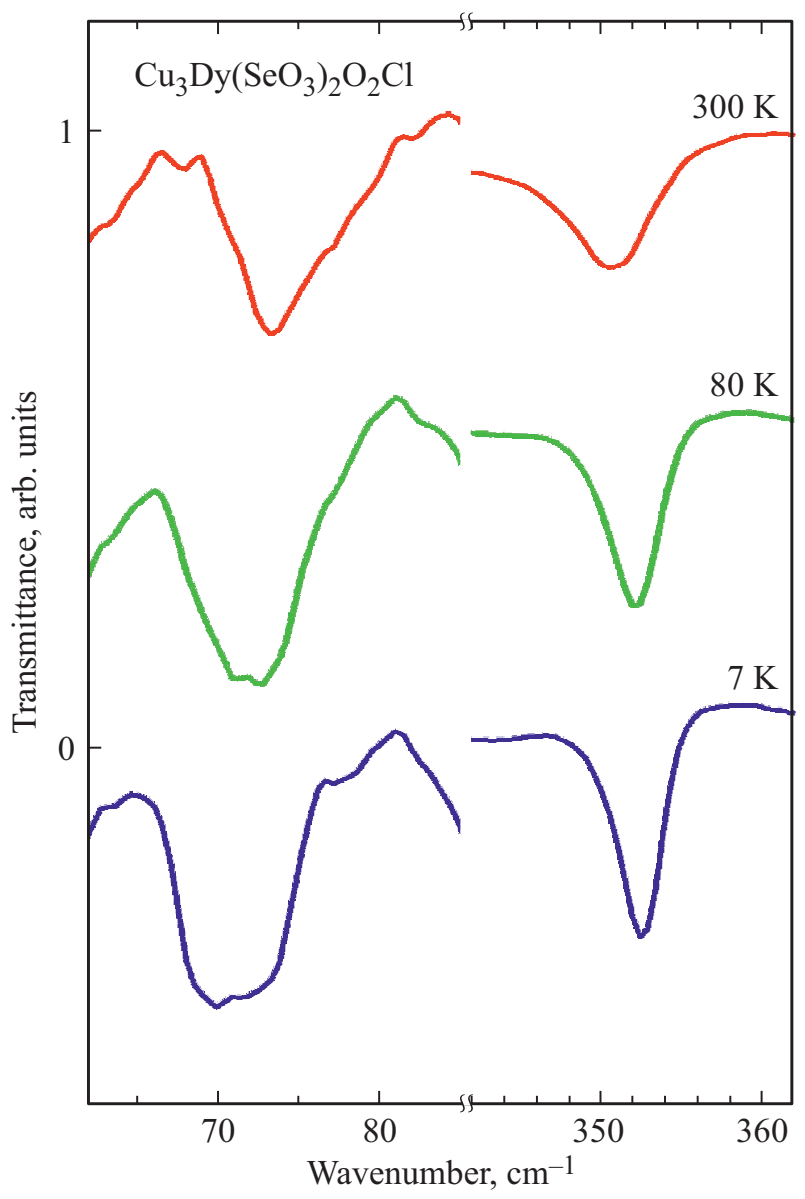

Рис. 8. Спектры диффузного пропускания поликристаллического $\mathrm{Cu}_{3} \mathrm{Dy}\left(\mathrm{SeO}_{3}\right)_{2} \mathrm{O}_{2} \mathrm{Cl}$ при трех различных температурах. Показаны два изолированных фонона в разных областях спектра.

частотном диапазоне с частотами выше $100 \mathrm{~cm}^{-1}$ ведут себя схожим образом: при понижении температуры их частоты растут, а полуширины уменьшаются.

Фононы с частотами ниже $100 \mathrm{~cm}^{-1}$ ведут себя подругому в зависимости от температуры. Во-первых, их частоты смягчаются при понижении температуры, вовторых, их полуширины слабо реагируют на изменение температуры, а для некоторых фононов наблюдается даже увеличение полуширины при охлаждении. Пример такого низкочастотного фонона вблизи $70 \mathrm{~cm}^{-1}$ показан на рис. 8.

Такое поведение является аномальным. Смягчение фонона, сопровождающееся уширением, можно рассматривать как развитие структурной нестабильности. В частности, в работе [14] при исследовании структурного фазового перехода в ферроборатах по результатам исследований методом ИК отражения было установлено, что полуширина фононов растет задолго до фазового перехода, являясь предвестником структурных изменений. Напомним, что родоначальник семейства, францисит $\mathrm{Cu}_{3} \mathrm{Bi}\left(\mathrm{SeO}_{3}\right)_{2} \mathrm{O}_{2} \mathrm{Cl}$, испытывает структурный фазовый переход, связываемый с подвижностью атомов 
галогена, в данном случае хлора, внутри гексагональных пространственных каналов. Мы полагаем, что в случае $\mathrm{Cu}_{3} \mathrm{Dy}\left(\mathrm{SeO}_{3}\right)_{2} \mathrm{O}_{2} \mathrm{Cl}$ структурный переход является виртуальным, нереализуемым при положительных температурах, а поведение низкочастотных фононов является указанием на развитие нестабильности в позициях атомов хлора. Альтернативным объяснением аномального поведения низкочастотных фононов могло бы быть взаимодействие решеточной и магнитной степеней свободы в кристалле $\mathrm{Cu}_{3} \mathrm{Dy}\left(\mathrm{SeO}_{3}\right)_{2} \mathrm{O}_{2} \mathrm{Cl}$. Франциситы являются низкоразмерными магнетиками, для которых наличие магнитных корреляций возможно при температурах, сильно превышающих температуру Нееля. Более того, в работе [40] найдены указания на возможное существование перехода Таулесса-Костерлица в семействе соединений со структурой францисита, в результате которого может образовываться коррелированная магнитная фаза со специфическими вихревыми возбуждениями. В результате взаимодействие низкочастотных фононов c магнитными корреляциями в парамагнитной фазе, а также с магнонами в антиферромагнитной фазе может также приводить к аномальному поведению фононов.

\section{Заключение}

Проведено систематическое исследование спектров $\mathrm{HПВО} \mathrm{серии} \mathrm{аналогов} \mathrm{францисита} \mathrm{Cu}_{3} R E\left(\mathrm{SeO}_{3}\right)_{2} \mathrm{O}_{2} \mathrm{Cl}$ $(R E$ - редкоземельный элемент, $R E=\mathrm{Nd}, \mathrm{Sm}, \mathrm{Eu}, \mathrm{Gd}$, $\mathrm{Dy}, \mathrm{Ho}, \mathrm{Tm}, \mathrm{Yb})$. Полученные зависимости поведения частот фононов от ионного радиуса укладываются в модель химического сжатия, при котором уменьшение параметров элементарной ячейки в серии изоструктурных соединений приводит к ужесточению фононов. Выявлены фононы, соответствующие колебаниям, в которых участвуют ионы РЗЭ. Для этих фононов наблюдается смягчение фононов за счет того, что влияние массы иона РЗЭ на частоту преобладает над эффектом химического сжатия. Для диспрозиевого соединения $\mathrm{Cu}_{3} \mathrm{Dy}\left(\mathrm{SeO}_{3}\right)_{2} \mathrm{O}_{2} \mathrm{Cl}$ обнаружено аномальное смягчение низкочастотных фононов, предположительно связанное со структурной нестабильностью в позициях атомов хлора.

\section{Финансирование работы}

Работа Н.Н.Н., В.А.Я. и С.А.К. (спектроскопические исследования) выполнена в ИСАН и поддержана РФФИ в рамках гранта № 19-02-00251, работа Е.С.К. (синтез соединений) выполнена в МГУ им. М.В. Ломоносова и поддержана РФФИ в рамках гранта № 19-33-60093 „Перспектива“.

\section{Конфликт интересов}

Авторы заявляют, что у них нет конфликта интересов.

\section{Список литературы}

[1] N.N. Kuzmin, S.A. Klimin, B.N. Mavrin, K.N. Boldyrev, V.A. Chernyshev, B.V. Mill, M.N. Popova. J. Phys. Chem. Solids, 138, 109266 (2018). DOI: 10.1016/j.jpcs.2019.109266

[2] N.N. Kuzmin, S.A. Klimin, B.N. Mavrin, K.N. Boldyrev, V.A. Chernyshev, B.V. Mill, M.N. Popova. Data in Brief, 28, 104889 (2020). DOI: 10.1016/j.dib.2019.104889

[3] S.A. Klimin, A.B. Kuzmenko, M.N. Popova, B.Z. Malkin, I.V. Telegina. Phys. Rev. B, 82, 174425-1 (2010). DOI: $10.1088 / 1742-6596 / 1389 / 1 / 012039$

[4] A.D. Molchanova, K.N. Boldyrev, M.N. Popova, M.A. Prosnikov, R.M. Dubrovin, V.Yu. Davydov., R.V. Pisarev. Phys. Rev. B, 96, 174305 (2017). DOI: 10.1103/PhysRevB.96.174305

[5] A.D. Molchanova, M.A. Prosnikov, V.P. Petrov, R.M. Dubrovin, S.G. Nefedov, D. Chernyshov, A.N. Smirnov, V.Yu. Davydov, K.N. Boldyrev, V.A. Chernyshev, R.V. Pisarev, M.N. Popova. J. All. Com., 865, 158797 (2021). DOI: $10.1016 /$ j.jallcom.2021.158797

[6] R.V. Pisarev, M.A. Prosnikov, V.Yu. Davydov, A.N. Smirnov, E.M. Roginskii, K.N. Boldyrev, A.D. Molchanova, M.N. Popova, M.B. Smirnov, V.Yu. Kazimirov. Phys. Rev. B, 93, 134306 (2016). DOI: 10.1103/PhysRevB.93.134306

[7] М.А. Просников, Р.М. Дубровин, А.Д. Молчанова, К.Н. Болдырев, А.Н. Смирнов, В.Ю. Давыдов, А.М. Балбашов, М.Н. Попова, Р.В. Писарев. ФТТ, 58 (12), 2427 (2016). [M.A. Prosnikov, R.M. Dubrovin, A.D. Molchanova, K.N. Boldyrev, A.N. Smirnov, V.Yu. Davydov, A.M. Balbashov, M.N. Popova, R.V. Pisarev. Phys. of Solid State, 58 (12), 2427 (2016). DOI: $10.21883 / \mathrm{ftt} .2016 .12 .43868 .239]$.

[8] K.N. Boldyrev, V.E. Anikeeva, O.I. Semenova, M.N. Popova. J. of Phys. Chem. C, $\mathbf{1 2 4}(42), 23307$ (2020). DOI: $10.1021 /$ acs.jpcc.0c06103

[9] D. Fausti, A. Nugroho, P. Loosdrecht, S.A. Klimin., M.N. Popova, L.N. Bezmaternykh. Phys. Rev. B, 74, 024403 (2006). DOI: 10.1103/PhysRevB.74.024403

[10] S.A. Klimin, M.N. Popova, B.N. Mavrin, P.H.M. Loosdrecht, L.E. Svistov, A.I. Smirnov, L.A. Prozorova, H.A. Krug von Nidda, Z. Seidov, A. Loidl, A.Ya. Shapiro, L.N. Demianets. Phys. Rev. B, 68, 174408-1 (2003). DOI: 10.1103/PhysRevB.68.174408

[11] M.N. Popova, A.B. Sushkov, S.A. Klimin, E.P. Chukalina, B.Z. Malkin, M. Isobe, Yu. Ueda. Phys. Rev. B, 65, 144303-1 (2002). DOI: 10.1103/PhysRevB.65.144303

[12] M.N. Popova, A.B. Sushkov, S.A. Golubchik, B.N. Mavrin, V.N. Denisov, B.Z. Malkin, A.I. Iskhakova, M. Isobe, Y. Ueda. ЖЭТФ, 115 (6), 2170 (1999) [M.N. Popova, A.B. Sushkov, S.A. Golubchik, B.N. Mavrin, V.N. Denisov, B.Z. Malkin, A.I. Iskhakova, M. Isobe, Y. Ueda. JETP, 88, 1186 (1999). DOI: $10.1134 / 1.558909]$.

[13] M.N. Popova, A.B. Sushkov, A.N. Vasil'ev, M. Isobe, Yu. Ueda. Письма в ЖЭТФ, 65, 711 (1997). [M.N. Popova, A.B. Sushkov, A.N. Vasil'ev, M. Isobe, Yu. Ueda. JETP Lett., 65, 743 (1997). DOI: 10.1134/1.567420].

[14] S.A. Klimin, A.B. Kuzmenko, M.A. Kashchenko, M.N. Popova. Phys. Rev. B, 93, 054304 (2016). DOI: 10.1103/PhysRevB.93.054304

[15] M.N. Popova, K.N. Boldyrev, S.A. Klimin, T.N. Stanislavchuk, A.A. Sirenko, L.N. Bezmaternykh. JMMM, 383, 250 (2015). DOI: $10.1016 /$ j.jmmm.2014.10.095. 
[16] M.N. Popova. EPJ Web of Conferences, 132, 01010 (2017). DOI: $10.1051 /$ epjconf/201713201010

[17] М.Н. Попова, К.Н. Болдырев. УФН, 189 (3), 292 (2019). [M.N. Popova, K.N. Boldyrev. Phys. Usp., 62 (3), 275 (2019). DOI: $10.3367 /$ UFNe.2018.06.038413].

[18] S.A. Klimin, E.A. Popova, M.N. Popova. J. of Physics: Conference Series, 1389, 012039 (2019). DOI: 10.1088/1742$6596 / 1389 / 1 / 012039$

[19] K.N. Boldyrev, T.N. Stanislavchuk, A.A. Sirenko, L.N. Bezmaternykh, M.N. Popova. Phys. Rev. B. Rapid Comm., 90, 121101(R) (2014). DOI: 10.1103/PhysRevB.90.121101

[20] К.Н. Болдырев, Б.Н. Маврин, М.Н. Попова, Л.Н. Безматерных. Опт. и спектр., $\mathbf{1 1 1}(3), 457$ (2011). [K.N. Boldyrev, B.N. Mavrin, M.N. Popova, L.N. Bezmaternykh. Opt. Spectrosc., 111 (3), 420 (2011). DOI: 10.1134/S0030400X11090049].

[21] R.V. Pisarev, K.N. Boldyrev, M.N. Popova, A.N. Smirnov, V.Yu. Davydov, L.N. Bezmaternykh, M.B. Smirnov, V.Yu. Kazimirov. Phys. Rev. B., 88, 024301 (2013). DOI: 10.1103/PhysRevB.88.024301

[22] E.A. Vinogradov, V.A. Yakovlev, N.N. Novikova, M.N. Popova, S.K. Saikin, B.Z. Malkin. Solid State Comm., 142 (5), 256 (2007). DOI: 10.1016/j.ssc.2007.02.032

[23] П.С. Бердоносов, В.А. Долгих. ЖНХ, 53 (9), 1451 (2008). [P.S. Berdonosov, V.A. Dolgikh. J. Inorg. Chem., 53 (9), 1353 (2008). DOI: 10.1134/S0036023608090027].

[24] P.S. Berdonosov, E.S. Kuznetsova, V.A. Dolgikh. Crystals, 8 (4), 159 (2018). DOI: 10.3390/cryst8040159

[25] K.V. Zakharov, E.A. Zvereva, P.S. Berdonosov, E.S. Kuznetsova, V.A. Dolgikh, L. Clark, C. Black, P. Lightfoot, W. Kockelmann, Z.V. Pchelkina, S.V. Streltsov, O.S. Volkova, A.N. Vasiliev. Phys. Rev. B, 90 (21), 214417 (2014). DOI: 10.1103/PhysRevB.90.214417

[26] S.A. Klimin, P.S. Berdonosov, E.S. Kuznetsova, Fiz. Nizk. Temp. 47 (12), 1119 (2021). DOI: 10.1063/10.0007075

[27] K.V. Zakharov, E.A. Zvereva, E.S. Kuznetsova, P.S. Berdonosov, V.A. Dolgikh, M.M. Markina, A.V. Olenev, A.A. Shakin, O.S. Volkova, A.N. Vasiliev. J. Alloys Compounds, 685, 442 (2016). DOI: 10.1016/j.jallcom.2016.05.213

[28] K.V. Zakharov, E.A. Zvereva, M.M. Markina, M.I. Stratan, E.S. Kuznetsova, S.F. Dunaev, P.S. Berdonosov, V.A. Dolgikh, A.V. Olenev, S.A. Klimin, L.S. Mazaev, M.A. Kashchenko, M.A. Ahmed, A. Banerjee, S. Bandyopadhyay, A. Iqbal, B. Rahaman, T. Saha-Dasgupta, A.N. Vasiliev. Phys. Rev. B, 94 (5), 054401 (2016). DOI: 10.1103/PhysRevB.94.054401

[29] M.M. Markina, K.V. Zakharov, E.A. Ovchenkov, P.S. Berdonosov, V.A. Dolgikh, E.S. Kuznetsova, A.V. Olenev, S.A. Klimin, M.A. Kashchenko, I.V. Budkin, I.V. Yatsyk, A.A. Demidov, E.A. Zvereva, A.N. Vasiliev. Phys. Rev. B, 96 (13), 134422 (2017). DOI: 10.1103/PhysRevB.96.134422

[30] С.А. Климин, Е.С. Кузнецова, П.С. Бердоносов. Опт. и спектр., 130 (1), 38 (2022). [S.A. Klimin, E.S. Kuznetsova, P.S. Berdonosov. Opt. Spectr., 130 (1), (2022), accepted]

[31] С.А. Климин, П.С. Бердоносов, Е.С. Кузнецова. Опт. и спектр., $\mathbf{1 2 9}(1), 46 \quad$ (2021). DOI: $\quad 10.21883 /$ OS.2021.01.50439.250-20 [S.A. Klimin, P.S. Berdonosov, E.S. Kuznetsova. Opt. Spectrosc., 129 (1), 47 (2021). DOI: 10.1134/S0030400X21010094].
[32] V. Gnezdilov, Yu. Pashkevich, P. Lemmens, V. Kurnosov, P. Berdonosov, V. Dolgikh, E. Kuznetsova, V. Pryadun, K. Zakharov, A. Vasiliev. Phys. Rev. B, 96 (11), 115144 (2017). DOI: 10.1103/PhysRevB.96.115144

[33] A. Pring, B.M. Gatehouse, W.D. Birch. Amer. Miner., 75 (11-12), 1421 (1990).

[34] P. Millet, B. Bastide, V. Pashchenko, S. Gnatchenko, V. Gapon, Y. Ksari, A. Stepanov. J. Mater. Chem., 11 (4), 1152 (2001). DOI: $10.1039 / \mathrm{b} 007920 \mathrm{k}$

[35] R. Becker, M. Johnsson. Solid State Sci., 7 (4), 375 (2005). DOI: $10.1016 /$ j.solidstatesciences.2004.10.045

[36] R. Berrigan, B.M. Gatehouse. Acta Crystallogr. C: Struct. Sci. Comm., 52, 496 (1996). DOI: 10.1107/S0108270195014120

[37] J.A. Mandarino. Eur. J. Miner., 6, 337 (1994).

[38] S.V. Krivovichev, G.L. Starova, S.K. Filatov. Miner. Magazine, 63 (2), 263 (1999). DOI: 10.1180/minmag.1999.063.2.12

[39] E.V. Nazarchuk, S.V. Krivovichev, O.Y. Pankratova, S.K. Filatov. Phys. Chem. Miner., 27, 440 (2000). DOI: $10.1007 / \mathrm{s} 002699900079$

[40] M. Pregelj, O. Zaharko, A. Gunther, A. Loidl, V. Tsurkan, S. Guerrero. Phys. Rev. B., 86 (14), 144409 (2012). DOI: $10.1103 /$ PhysRevB.86.144409

[41] S.A. Nikolaev, V.V. Mazurenko, A.A. Tsirlin, V.G. Mazurenko. Phys. Rev. B., 94 (14), 144412 (2016). DOI: 10.1103/PhysRevB.94.144412

[42] I. Rousochatzakis, J. Richter, R. Zinke, A.A. Tsirlin. Phys. Rev. B., 91, 024416 (2015). DOI: 10.1103/PhysRevB.91.024416

[43] М.М. Маркина, П.С. Бердоносов, В.А. Долгих, К.В. Захаров, Е.С. Кузнецова, А.Н. Васильев. УФН, 191, 358 (2021). DOI: 10.3367/UFNr.2020.05.038773 [M.M. Markina, P.S. Berdonosov, V.A. Dolgikh, K.V. Zakharov, E.S. Kuznetsova, A.N. Vasil'ev. Phys. Usp., 64, 344 (2021). DOI: 10.3367/UFNe.2020.05.038773]

[44] С.А. Климин, М.С. Радионов, В.А. Яковлев, Н.Н. Новикова, А.В. Песчанский. Опт. и спектр., 129 (1), 46 (2021). DOI: 10.21883/OS.2021.01.50438.24920 [S.A. Klimin, M.S. Radionov, V.A. Yakovlev, N.N. Novikova, A.V. Peschanskii. Opt. Spectr., 129, 42 (2021). DOI: 10.1134/S0030400X21010082]

[45] R. Yankova, S. Genieva, N. Halachev, G. Dimitrova. J. Mol. Struct., 1106, 82 (2016). DOI: 10.1016/j.molstruc.2015.10.091

[46] A. Bindu Gopinath, S. Devanarayanan. Indian J. Phys., 72B (1), 75 (1998).

[47] Z. Mička, M. Daněk, J. Loub, B. Strauch, J. Podlanová, J. Hašek. J. Solid State Chem., $77(2), 306$ (1988). DOI: 10.1016/0022-4596(88)90253-8

[48] K.H. Miller, P.W. Stephens, C. Martin, E. Constable, R.A. Lewis, H. Berger, G.L. Carr, D.B. Tanner. Phys. Rev. B., 86, 174104 (2012). DOI: 10.1103/PhysRevB.86.174104

[49] W. Theiß. Surf. Sci. Reports., 29(3-4), 91 (1997). DOI: 10.1016/S0167-5729(96)00012-X

[50] G. Herzberg. Molecular Spectra and Molecular Structure. Part II. Infrared and Raman Spectra of Polyatomic Molecules (Van Nostrand Comp., New York, 1945)

[51] R.D. Shannon. Acta Crystallogr. A., 32, 751 (1976). DOI: $10.1107 / \mathrm{S} 0567739476001551$

[52] Database of Ionic Radii [электронный pecypc]. URL: http://abulafia.mt.ic.ac.uk/shannon/ptable.php 